\title{
Differential expression of histone cluster 3, H2a in triple negative breast cancer.
}

Shahan Mamoor, $\mathrm{MS}^{1}$

${ }^{1}$ shahanmamoor@gmail.com

East Islip, NY 11730

Women diagnosed with triple negative breast cancer are predicted to benefit neither from endocrine therapy nor from HER2-targeted therapies ${ }^{1}$. We mined published microarray datasets ${ }^{2,3}$ to determine in an unbiased fashion and at the systems level genes most differentially expressed in the primary tumors of patients with breast cancer. We report here significant differential expression of the gene encoding histone cluster 3, H2a, HIST3H2A, when comparing the tumor cells of patients with triple negative breast cancer to normal mammary ductal cells ${ }^{2}$. HIST3H2A was also differentially expressed in bulk tumor in human breast cancer ${ }^{3}$. HIST3H2A mRNA was present at significantly increased quantities in TNBC tumor cells relative to normal mammary ductal cells. Analysis of human survival data revealed that expression of HIST3H2A in primary tumors of the breast was correlated with recurrence-free survival in patients with luminal A and HER2+ type cancer, while within triple negative breast cancer, primary tumor expression of HIST3H2A was correlated with distant metastasis-free survival in patients with immunomodulatory, mesenchymal, and luminal androgen receptor subtype disease. HIST3H2A may be of relevance to initiation, maintenance or progression of triple negative breast cancers.

Keywords: breast cancer, HIST3H2A, histone cluster 3, H2a, systems biology of breast cancer, targeted therapeutics in breast cancer. 
Patients with hormone receptor positive disease can benefit from endocrine therapy ${ }^{4}$ : aromatase inhibition such as that provided by exemestane or letrozole, or estrogen receptor antagonism such as that provided by tamoxifen or fulvestrant. Patients whose tumors overexpress the human epidermal growth factor 2 (HER2) can be treated with HER2-targeted therapies: HER2 inhibitors, immunoglobulin ${ }^{5}$ - or small molecule-based ${ }^{6}$. As triple negative breast cancer (TNBC) is defined by its lack of HER2 expression as well as by its lack of expression of the hormone receptor estrogen, patients diagnosed with triple negative breast cancer are suggested to benefit from neither of these treatment strategies.

Understanding the transcriptional composition of triple negative breast cancers in humans can facilitate development of novel therapeutics and contribute to efforts to prevent progression to metastatic stages. We mined published microarray data ${ }^{2,3}$ to understand at the transcriptome level and in an unbiased fashion genes most differentially expressed in the tumor cells of patients with triple negative breast cancer.

\section{Methods}

We utilized datasets GSE38959 ${ }^{2}$ and GSE109169 ${ }^{3}$ for this differential gene expression analysis of female breast cancer in conjunction with GEO2R. GSE38959 was generated using Agilent-014850 Whole Human Genome Microarray 4x44K G4112F technology with $n=13$ samples of normal mammary gland ductal cells and $n=30$ samples of tumor cells from patients with triple negative breast cancer; analysis was performed using platform GPL4133. GSE109169 was generated using Affymetrix Human Exon 1.0 ST Array technology with $n=25$ normal breast tissue and $n=25$ tumors of the breast; analysis was performed using platform GPL5175. The tissues from this dataset are paired tissues (25 tumors matching 25 normal breast tissues from 25 patients). The Benjamini and Hochberg method of $p$-value adjustment was used for ranking of differential expression but raw $p$-values were used to assess statistical significance of global differential expression. Log-transformation of data was auto-detected, and the NCBI-generated category of platform annotation was used. A statistical test was performed to evaluate whether HIST3H2A expression was significantly different between primary breast tumors and breast tissue using a two-tailed t-test.

For Kaplan-Meier survival analysis, we used the Kaplan-Meier plotter tool ${ }^{7}$ for correlation of HIST3H2A mRNA expression levels with recurrence-free survival (RFS) in $n=431$ patients with basal subtype cancer, $n=596$ patients with luminal A subtype cancer, $n=439$ patients with luminal B subtype cancer, $n=362$ patients with HER $2+$ cancer, and $n=51$ patients with normal-like subtype cancer. In patients with triple negative breast cancer $^{7}$, we analyzed distant metastasis-free survival (DMFS) for $n=171$ patients with immunomodulatory subtype TNBC, $n=71$ patients with basal-like 2 subtype TNBC, $n=219$ patients with immunomodulatory subtype TNBC, $n=138$ patients with luminal androgen receptor subtype TNBC, $n=130$ patients with mesenchymal subtype TNBC, and $n=39$ patients with mesenchymal stem-like subtype TNBC.

\section{Results}

We performed discovery of genes associated with triple negative breast cancer in humans by mining two independently published microarray datasets ${ }^{2,3}$.

\section{HIST3H2A is differentially expressed in primary tumors and tumor cells of the breast.}

Comparison of the tumor cells of patients with triple negative breast cancer to normal mammary ductal cells ${ }^{2}$ revealed histone cluster 3, H2a, encoded by HIST3H2A, as among the genes whose expression was most significantly different in human TNBC as compared to the normal breast (Chart 1). When sorting each of the genes expressed in the tumor cells of patients with triple negative breast cancer based on significance of difference as compared to normal mammary ductal cells, HIST3H2A ranked 210 out of 45015 total transcripts, equating to $99.5 \%$ differential expression (Chart 1). Differential expression of HIST3H2A in the tumor cells of patients with triple negative breast cancer was statistically significant (Chart 1; $p=3.21 \mathrm{E}-11$ ). 
Analysis of a second microarray datase ${ }^{3}$, here comparing 25 normal breast tissues to 25 tumors of the breast revealed that HIST3H2A was also among the genes most differentially expressed in human breast cancer, here in the tumors of women diagnosed with early-onset breast cancer (Chart 2). When sorting each of the genes expressed in tumors of the breast based on significance of difference as compared to normal breast tissue, HIST3H2A ranked 4843 out of 19076 total transcripts, equating to $74.6 \%$ differential expression (Chart 2). Differential expression of HIST3H2A in female breast cancer was statistically significant (Chart $2 ; p=4.32 \mathrm{E}-04)$.

These data indicated that differential expression of HIST3H2A was not an artifact of a single microarray dataset, was not an artifact of FACS isolation, and suggested that perturbed expression of HIST3H2A was likely a transcriptional feature of triple negative breast cancers and early-onset human breast cancers.

HIST3H2A is expressed at significantly higher levels in triple negative tumor cells as compared to normal cells of the breast.

We obtained exact mRNA expression levels for HIST3H2A - from normal mammary ductal cells of the breast and from tumor cells of patients with triple negative breast cancer - to understand the magnitude and direction of HIST3H2A expression change in TNBC. HIST3H2A mRNA transcript was present at higher levels in triple negative tumor cells of the breast as compared to normal mammary ductal cells (Figure 1). Increased expression of HIST3H2A in primary tumor-isolated triple negative breast cancer cells was statistically significant (Figure 1: $p<0.0001$ ). HIST3H2A was expressed at 1073.5 \pm 761.7 arbitrary units (A.U.) in normal mammary ductal cells, while it was expressed at $4164.7 \pm 1703.9$ A.U. in tumor cells of patients with triple negative breast cancer. We calculated a mean fold change of 3.88 when comparing triple negative breast cancer cells to normal mammary ductal cells.

\section{HIST3H2A expression correlates with survival outcomes in luminal A and HER2+ human breast cancer.}

We performed Kaplan-Meier survival analysis to evaluate potential relationships between primary tumor HIST3H2A mRNA expression levels and survival outcomes in patients with breast cancer, when analyzing outcomes based on the molecular subtype of the patient (PAM50 classification): luminal A, luminal B, HER2+, normal-like, and basal-like. We observed a statistically significant correlation between HIST3H2A expression and recurrence-free survival (RFS) in patients with luminal A subtype breast cancer (Figure 2; log rank $p$-value: 0.031 for recurrence-free survival, hazard ratio: 0.63 (0.410.96) (Fig. 2)). HIST3H2A mRNA levels were a positive prognostic indicator in luminal A breast cancer patients. Median RFS was 169.2 months for luminal A patients with low tumor expression of HIST3H2A while median RFS was 175.46 months for luminal A patients with high tumor expression of HIST3H2A (Chart 3).

We observed a correlation between HIST3H2A expression and recurrence-free survival (RFS) in patients with HER2+ subtype breast cancer which trended towards statistical significance (Figure 2; $\log$ rank $p$-value: 0.075 for recurrence-free survival, hazard ratio: 1.42 (0.96-2.1) (Fig. 2)). HIST3H2A mRNA levels were a negative prognostic indicator in HER2+ breast cancer patients. Median RFS was 56.64 months for HER2+ patients with low tumor expression of HIST3H2A while median RFS was 42.87 months for HER2+ patients with high tumor expression of HIST3H2A (Chart 3).

HIST3H2A primary tumor expression was not correlated with recurrence-free survival in basal subtype (Figure 2; log rank $p$-value: 0.5 for RFS, hazard ratio: 1.14 (0.78-1.67) (Fig. 2), luminal B subtype (Figure 2; log rank $p$-value: 0.68 for RFS, hazard ratio: 0.93 (0.66-1.32) (Fig. 2)), or in the normal-like subtype (Figure 2; log rank $p$-value: 0.34 for RFS, hazard ratio: 0.61 (0.22-1.68) (Fig. 2)). 
Influence of primary tumor HIST3H2A expression on survival outcomes based on TNBC molecular subtype.

We also performed Kaplan-Meier survival analysis to examine relationships between HIST3H2A mRNA expression levels with survival strictly in patients with triple negative breast cancer, analyzing outcomes based on the TNBC molecular subtype ${ }^{8}$ of the patient: immunomodulatory, basal-like 2, immunomodulatory, mesenchymal, mesenchymal stem-like, and luminal androgen receptor. We observed a correlation between HIST3H2A expression and distant metastasis-free survival (DMFS) in TNBC patients with immunomodulatory subtype disease which approached statistical significance (Figure $3 ; \log$ rank $p$-value: 0.064 for distant metastasis-free survival, hazard ratio: 1.68 (0.96-2.92) (Fig. 3)). HIST3H2A mRNA levels were a negative prognostic indicator in patients with immunomodulatory TNBC. Median DMFS was 102.61 months for immunomodulatory TNBC patients with low tumor expression of HIST3H2A while median DMFS was 35.42 months for immunomodulatory TNBC patients with high tumor expression of HIST3H2A (Chart 4).

We also observed a correlation between HIST3H2A expression and distant metastasis-free survival (DMFS) in TNBC patients with mesenchymal subtype disease which trended towards statistical significance (Figure 3; log rank $p$-value: 0.098 for distant metastasis-free survival, hazard ratio: 1.66 (0.93.05) (Fig. 3)). HIST3H2A mRNA levels were a negative prognostic indicator in patients with mesenchymal TNBC. Median DMFS was 30.62 months for mesenchymal TNBC patients with low tumor expression of HIST3H2A while median DMFS was 25 months for mesenchymal TNBC patients with high tumor expression of HIST3H2A (Chart 4).

We also observed a statistically significant correlation between HIST3H2A expression and distant metastasis-free survival (DMFS) in TNBC patients with luminal androgen receptor subtype disease (Figure 3; log rank $p$-value: 0.029 for distant metastasis-free survival, hazard ratio: 1.92 (1.063.48) (Fig. 3)). HIST3H2A mRNA levels were a negative prognostic indicator in patients with luminal androgen receptor TNBC. Median DMFS was 72.2 months for luminal androgen receptor TNBC patients with low tumor expression of HIST3H2A while median DMFS was 23.49 months for luminal androgen receptor TNBC patients with high tumor expression of HIST3H2A (Chart 4).

HIST3H2A primary tumor expression was not correlated with distant metastasis-free survival in basal-like 1 subtype (Figure 3; log rank $p$-value: 0.1 for DMFS, hazard ratio: $1.6(0.9-2.85)$ (Fig. 3), basal-like 2 subtype (Figure 3; log rank $p$-value: 0.6 for DMFS, hazard ratio: 1.22 (0.58-2.61) (Fig. 3)), and in the mesenchymal stem-like subtype (Figure 3; log rank $p$-value: 0.73 for DMFS, hazard ratio: 1.2 (0.43-3.3) (Fig. 3)).

\section{Discussion}

We performed comparative transcriptome analysis using two independently published microarray datasets $^{2,3}$ to define and catalog transcriptional differences in human triple negative breast cancer. We provided evidence here that differential expression of histone cluster $3, \mathrm{H} 2 \mathrm{a}$, encoded by the HIST3H2A gene, is a defining transcriptional feature of triple negative breast cancer. HIST3H2A was also differentially expressed in the bulk tumors of patients with early-onset breast cancer. Expression of HIST3H2A was correlated with recurrence-free survival in patients with luminal A and HER2+ subtype breast cancer, and within triple negative breast cancer patients, primary tumor expression of HIST3H2A was correlated with distant metastasis-free survival in patients with immunomodulatory, mesenchymal, and luminal androgen receptor subtype disease. Histone cluster $3, \mathrm{H} 2 \mathrm{a}$ and the molecular processes to which it pertains to may be relevant to the initiation or progression of triple negative breast cancer in humans. 


\section{References}

1. Foulkes, W.D., Smith, I.E. and Reis-Filho, J.S., 2010. Triple-negative breast cancer. New England journal of medicine, 363(20), pp.1938-1948.

2. Komatsu, M., Yoshimaru, T., Matsuo, T., Kiyotani, K., Miyoshi, Y., Tanahashi, T., Rokutan, K., Yamaguchi, R., Saito, A., Imoto, S. and Miyano, S., 2013. Molecular features of triple negative breast cancer cells by genome-wide gene expression profiling analysis. International journal of oncology, 42(2), pp.478-506.

3. Chang, J.W., Kuo, W.H., Lin, C.M., Chen, W.L., Chan, S.H., Chiu, M.F., Chang, I.S., Jiang, S.S., Tsai, F.Y., Chen, C.H. and Huang, P.H., 2018. Wild-type p53 upregulates an early onset breast cancerassociated gene GAS7 to suppress metastasis via GAS7-CYFIP1- mediated signaling pathway. Oncogene, 37(30), pp.4137-4150.

4. Spring, L.M., Gupta, A., Reynolds, K.L., Gadd, M.A., Ellisen, L.W., Isakoff, S.J., Moy, B. and Bardia, A., 2016. Neoadjuvant endocrine therapy for estrogen receptor-positive breast cancer: a systematic review and meta-analysis. JAMA oncology, 2(11), pp.1477-1486.

5. Slamon, D., Eiermann, W., Robert, N., Pienkowski, T., Martin, M., Press, M., Mackey, J., Glaspy, J., Chan, A., Pawlicki, M. and Pinter, T., 2011. Adjuvant trastuzumab in HER2-positive breast cancer. New England journal of medicine, 365(14), pp.1273-1283.

6. Park, J.W., Liu, M.C., Yee, D., Yau, C., van’t Veer, L.J., Symmans, W.F., Paoloni, M., Perlmutter, J., Hylton, N.M., Hogarth, M. and DeMichele, A., 2016. Adaptive randomization of neratinib in early breast cancer. New England Journal of Medicine, 375(1), pp.11-22.

7. Györffy, B., Lanczky, A., Eklund, A.C., Denkert, C., Budczies, J., Li, Q. and Szallasi, Z., 2010. An online survival analysis tool to rapidly assess the effect of 22,277 genes on breast cancer prognosis using microarray data of 1,809 patients. Breast cancer research and treatment, 123(3), pp.725-731.

8. Lehmann, B.D., Bauer, J.A., Chen, X., Sanders, M.E., Chakravarthy, A.B., Shyr, Y. and Pietenpol, J.A., 2011. Identification of human triple-negative breast cancer subtypes and preclinical models for selection of targeted therapies. The Journal of clinical investigation, 121(7), pp.2750-2767. 
Rank: 210

Probe ID: 7742

p-value: $3.21 \mathrm{E}-11$

$3 \quad$ t: -8.7059458

B: 15.473623

4 Gene: HIST3H2A

Gene name: histone cluster 3, $\mathrm{H} 2 \mathrm{a}$

\section{Chart 1: HIST3H2A is differentially expressed in triple negative breast cancer.}

Rank of differential expression, probe ID, $\mathrm{p}$-value of global differential expression, $\mathrm{t}$, a moderated tstatistic, B, the log-odds of differential expression between the groups compared, gene and gene name are listed in this chart. 
Rank: 4843

Probe ID: 2459616

$p$-value: $4.32 \mathrm{E}-04$

$3 \quad$ t: -3.76

4

B: -0.672399

Gene: HIST3H2A

Gene name: histone cluster 3, $\mathrm{H} 2 \mathrm{a}$

Chart 2: HIST3H2A is differentially expressed in early-onset human breast cancer.

7 Rank of differential expression, probe ID, p-value of global differential expression, $t$, a moderated tstatistic, B, the log-odds of differential expression between the groups compared, gene and gene name are listed in this chart. 


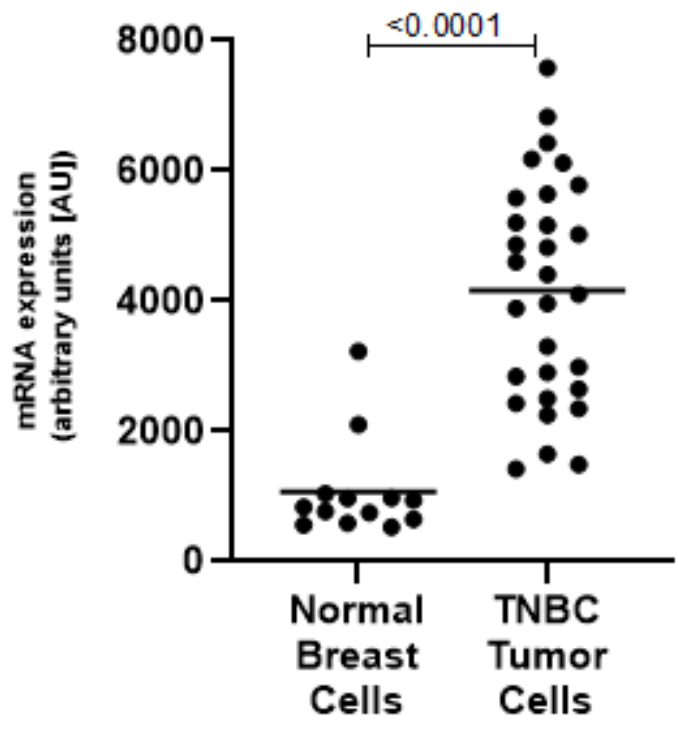

Figure 1: HIST3H2A is expressed at significantly higher levels in primary TNBC tumor cells as compared to normal cells of the breast.

The mRNA expression level of HIST3H2A in normal mammary ductal cells (left) and in the isolated primary tumor cells of patients with triple negative breast cancer (right) is graphically depicted with the result of a statistical test evaluating significance of difference in HIST3H2A expression between normal cells of the breast and TNBC tumor cells, a $p$-value, listed above. 


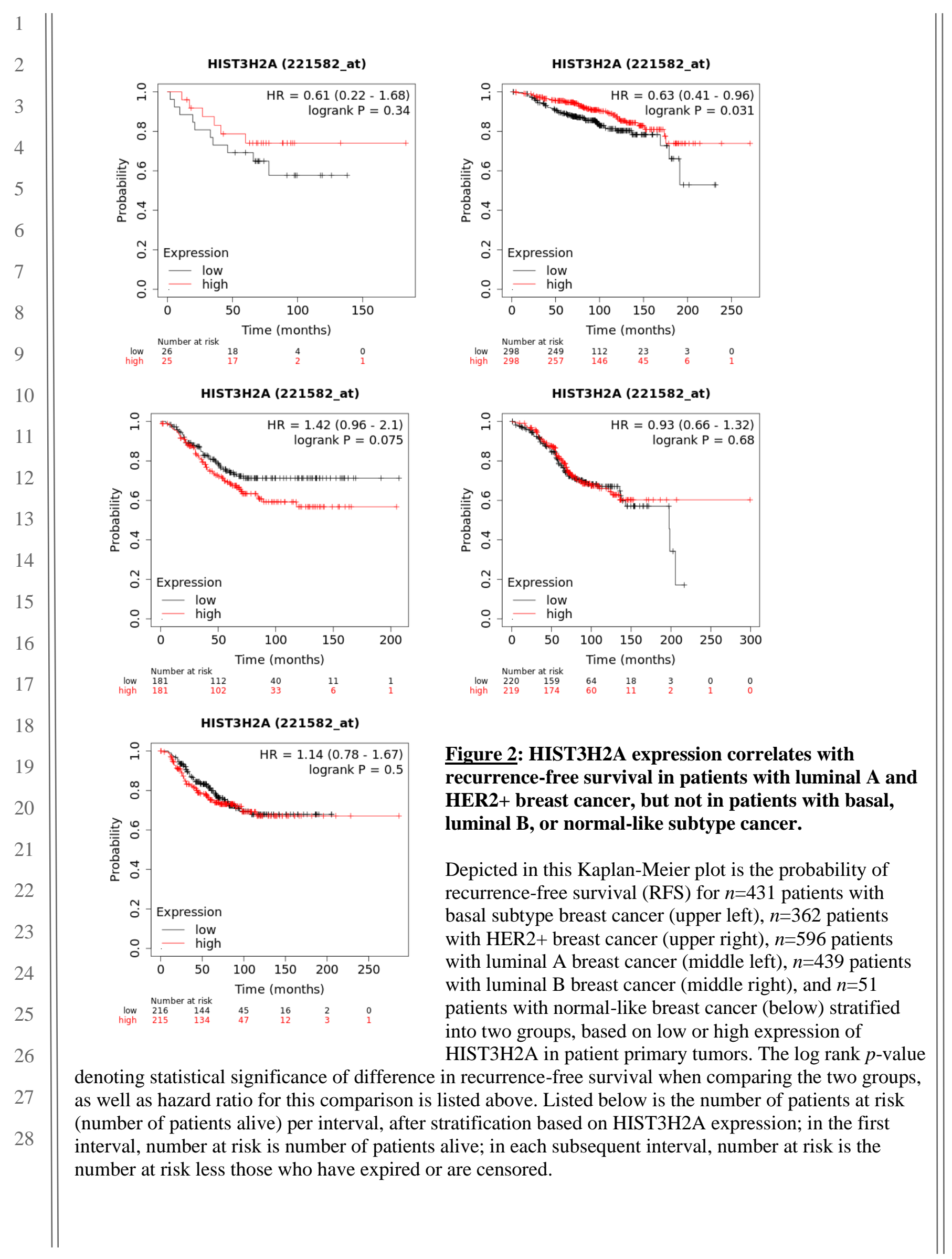


Low HIST3H2A expression: 169.2 months

High HIST3H2A expression: 175.46 months

Chart 3: Median recurrence-free survival is superior in luminal A subtype breast cancer patients with high primary tumor expression of HIST3H2A.

The recurrence-free survival of $n=596$ luminal A subtype breast cancer patients based on stratification into low or high tumor expression of HIST3H2A is listed in this chart.

Low HIST3H2A expression: 56.64 months

Chart 3: Median recurrence-free survival is superior in HER2+ subtype breast cancer patients with low primary tumor expression of HIST3H2A.

The recurrence-free survival of $n=362$ HER $2+$ subtype breast cancer patients based on stratification into low or high tumor expression of HIST3H2A is listed in this chart. 

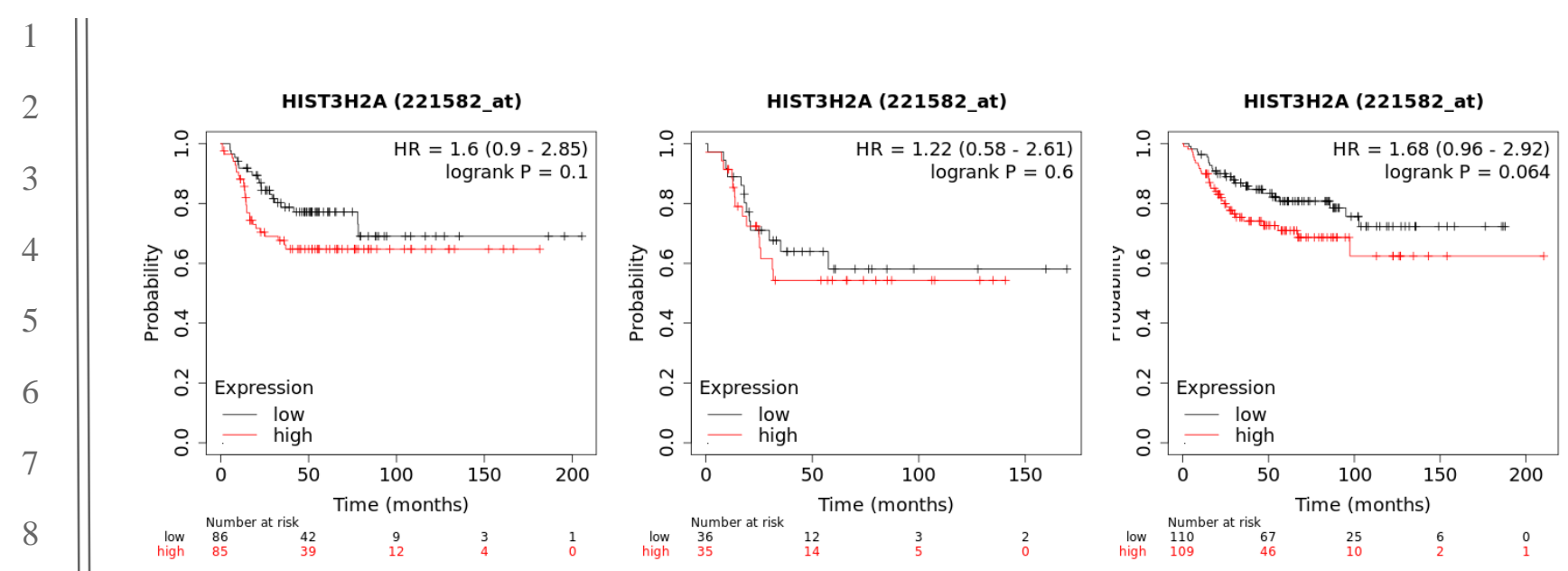

Figure 3: HIST3H2A expression correlates with distant metastasis-free survival in triple negative breast cancer patients whose tumors are of the immunomodulatory, mesenchymal, and luminal androgen receptor molecular subtype.

Depicted in this Kaplan-Meier plot is the probability of distant metastasis-free survival (DMFS) for $n=171$ patients with basal-like 1 subtype TNBC (upper left), $n=71$ patients with basal-like 2 subtype TNBC (upper right), $n=219$ patients with immunomodulatory subtype TNBC (middle left), $n=138$ patients with luminal androgen receptor subtype TNBC (middle right), $n=130$ patients with mesenchymal subtype TNBC (lower left), and $n=39$ patients with mesenchymal stem-like subtype TNBC (lower right), stratified into two groups, based on low or high expression of HIST3H2A in the primary tumors of patients with TNBC. The log rank p-value denoting statistical significance of difference in distant metastasis-free survival when comparing the two groups, as well as hazard ratio for this comparison is listed above. Listed below is the number of patients at risk (number of patients alive) per interval, after stratification based on HIST3H2A expression; in the first interval, number at risk is number of patients alive; in each subsequent interval, number at risk is the number at risk less those who have expired or are censored. 


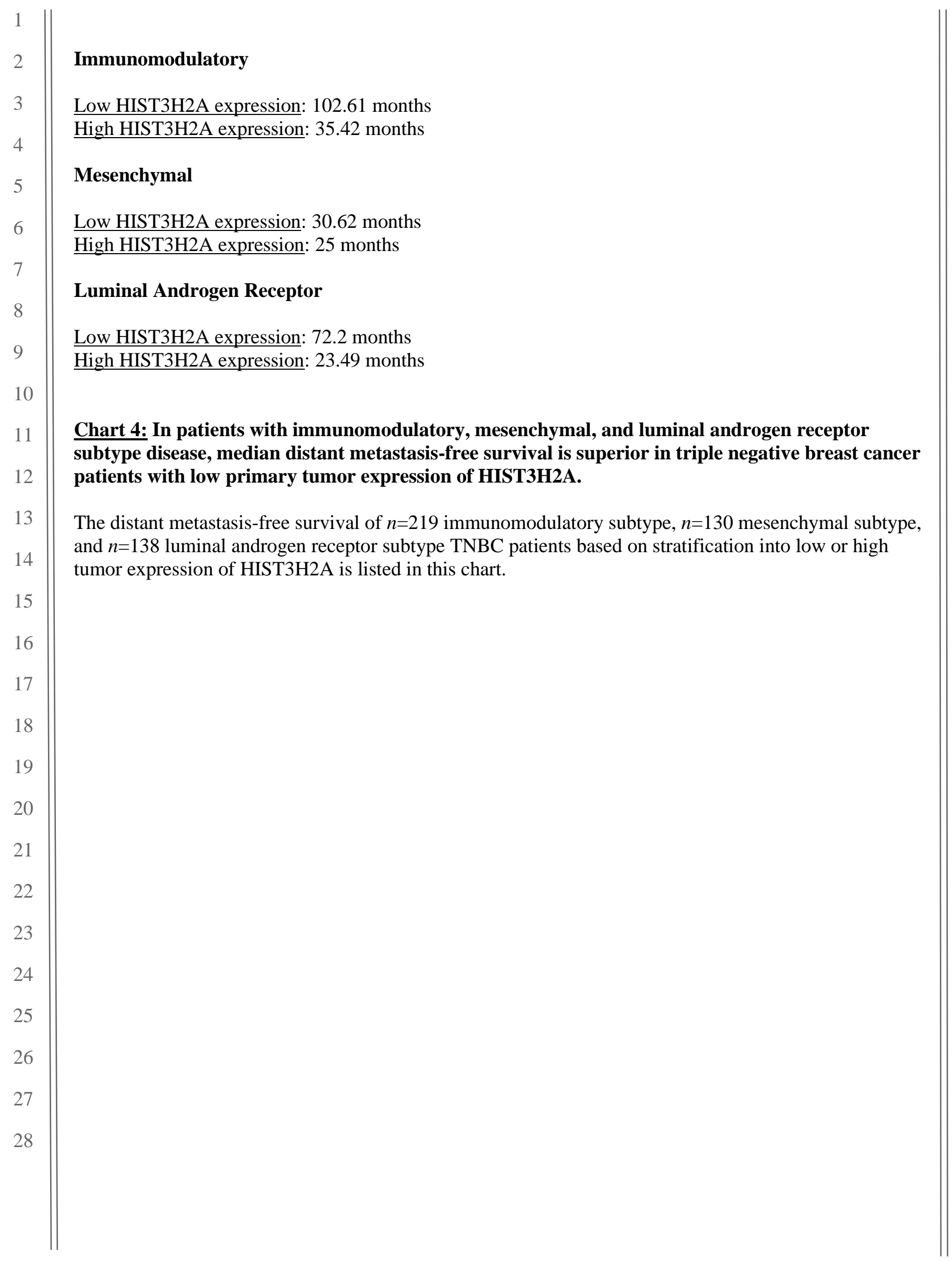

